\title{
Correlación entre Sodio, Cloro y Potasio del Cuerpo Vítreo y el Intervalo Postmortem sobre los 2.850 msnm
}

\author{
Correlation Between Sodium, Chlorine and Potassium of the \\ Vitreous Body and the Postmortem Interval Above $2.850 \mathrm{msnm}$
}

Clivia Guerrero-Urbina ${ }^{1,2,3}$; Martha Fors ${ }^{4}$; Mariano del Sol ${ }^{1,5}$ \& Gabriel M. Fonseca ${ }^{1,6}$

GUERRERO-URBINA, C.; FORS, M.; DEL SOL, M. \& FONSECA, G. M. Correlación entre sodio, cloro y potasio del cuerpo vítreo y el intervalo postmortem sobre los 2.850 msnm. Int. J. Morphol., 39(2):554-559, 2021.

RESUMEN: El intervalo Postmortem (IPM) es un importante desafío por resolver en la patología forense, y consiste en poder determinar el tiempo transcurrido desde la muerte hasta el momento de la autopsia. Dada la poca confiabilidad de algunos métodos por la gran influencia de factores externos y propios del cadáver, la bioquímica forense ha recibido considerable atención por sus niveles de seguridad. La ciudad de Quito se ubica en la cordillera de Los Andes a $2850 \mathrm{msnm}$, sin embargo, en la literatura no existen reportes de medición de estos parámetros a una altura como ésta. El objetivo fue establecer una correlación entre sodio $\left(\mathrm{Na}^{+}\right)$, cloro $(\mathrm{Cl}-)$ y potasio $(\mathrm{K}+)$ del humor vítreo del cuerpo vítreo y el IPM a la altura de la ciudad de Quito. Para el estudio se utilizaron 128 muestras de cuerpo vítreo provenientes de 16 autopsias practicadas en la Unidad de Medicina Legal Zona 9, en IPM de 6, 12, 24, 48, 72, 96, 120 y 144 horas. Para la obtención del humor vítreo se siguió el método convencional, se dividió para las 8 alícuotas y se llevó a congelación -20 ${ }^{\circ} \mathrm{C}$ hasta el momento de su análisis. La cuantificación de la concentración de electrolitos $\mathrm{Na}^{+}, \mathrm{Cl}^{-}$y Potasio ${ }^{+}$se realizó mediante analizador ISE de Roche Cobas (Roche Diagnostics) C501, calibrado para uroanálisis, y no fue necesaria la dilución. Durante la calibración y cada tres muestras, se midió un estándar interno para corregir los efectos de la desviación sistemática en la calibración. El enfoque estadístico se basó en un análisis de correlación lineal, utilizando el coeficiente de correlación de Spearman. La correlación entre las horas postmortem y las concentraciones de los diferentes electrolitos, fueron estadísticamente significativas. Se pudo corroborar una correlación lineal significativa entre el IPM y el aumento del $\mathrm{K}^{+}$en el HV.

PALABRAS CLAVE: Electrolitos; Intervalo postmortem; Cuerpo vítreo.

\section{INTRODUCCIÓN}

El intervalo postmortem (IPM) es una de las problemáticas que enfrenta la práctica forense (Li et al., 2018), y equivale al tiempo que ha transcurrido después de que un ser humano ha muerto (Chandrakanth et al., 2013; Yang et al., 2018) hasta el tiempo de examinación postmortem (Jashnani et al., 2010). Las investigaciones del IPM determinan más confiabilidad en periodos postmortem temprano (Gerometta et al., 2019) que en postmortem tardíos (Donaldson \& Lamont, 2014).

En la actualidad la estimación del tiempo de muerte se basa en el enfriamiento corporal, lividez postmortem, y datación por radiocarbono, que son procesos predominan- temente físicos. Rigor mortis y reagibilidad supravital del músculo esquelético (McCleskey et al., 2016) son procesos fisicoquímicos; la putrefacción se basa principalmente en procesos bacterianos (Tagliaro et al., 1999). Los métodos químicos se fundamentan en procesos metabólicos, autolisis y difusión, de acuerdo con el gradiente de concentración y la entomología (Li et al., 2018). La química permite medir los cambios de concentraciones en fluidos de varios compartimientos (Thierauf et al., 2009). La autolisis produce aumento de potasio, disminución de sodio y cloro que avanza rápidamente en la sangre y líquido cerebroespinal; sin embargo, en el cuerpo vítreo transcurre lentamente debido a su topografía aislada y confinada (Madea, 2016).

\footnotetext{
${ }^{1}$ Programa de Doctorado en Ciencias Morfológicas, Facultad de Medicina, Universidad de La Frontera, Temuco, Chile.

${ }^{2}$ Carrera de Obstetricia, Facultad de Ciencias Médicas, Universidad Central del Ecuador, Quito, Ecuador.

${ }^{3}$ Unidad de Medicina Legal, Zona 9. Policía Nacional del Ecuador, Quito, Ecuador.

${ }^{4}$ Universidad de Las Américas, Facultad de Ciencias de la Salud, Escuela de Medicina, Quito, Ecuador.

${ }^{5}$ Centro de Excelencia en Estudios Morfológicos y Quirúrgicos, Universidad de La Frontera, Temuco, Chile.

${ }^{6}$ Centro de Investigación en Odontología Legal y Forense (CIO), Facultad de Odontología, Universidad de La Frontera, Temuco, Chile.
} 
La bioquímica postmortem es importante para la toxicología forense, ya que proporciona información para determinar la causa de muerte y el IPM mediante estudios realizados en fluidos como en el cuerpo vítreo, que es acelular y está aislado siendo menos susceptible a la contaminación y a cambios bioquímicos (Foster et al., 2016). La función, la anatomía y la composición química del cuerpo vítreo permite a la toxicología y la química (Montefusco-Pereira \& Pinto, 2016) estimar el IPM (Zhou et al., 2007).

El segmento anterior del bulbo ocular presenta dos cámaras, anterior y posterior. En el segmento posterior del bulbo ocular en la cámara póstrema del bulbo ocular se encuentra el cuerpo vítreo. El cuerpo vítreo presenta la fosa hialoidea, canal hialoideo, membrana vítrea y humor vítreo.

El cuerpo vítreo o extremo posterior, es la cámara más grande y se forma durante la vida embrionaria entre el lente y la retina, y contiene células fagocíticas que eliminan los detritus y mantienen la visión (Montefusco-Pereira \& Pinto, 2016). El fluido vítreo es un gel claro viscoelástico suave (Mulla et al., 2005) que ocupa la cavidad vítrea o cavidad posterior del bulbo ocular y cuyas funciones son las de dar volumen al bulbo ocular, sostener la retina y mantener su transferencia de manera que los haces de luz puedan atravesarla (Moore \& Dalley, 2013). La superficie uniforme permite la recepción de imágenes nítidas (MontefuscoPereira \& Pinto, 2016), su peso aproximado es de $4 \mathrm{~g}$ y ocupa un volumen de $4 \mathrm{ml}$ que varía con la edad y el tamaño del bulbo ocular (Thierauf et al., 2009; Gerometta et al., 2019).

La composición del cuerpo vítreo está basada en un $98 \%$ de agua, siendo el ácido hialurónico el principal glucosaminoglicano, además de fibras de colágeno, los componentes inorgánicos son el $\mathrm{Na}^{+}, \mathrm{K}^{+}, \mathrm{Ca}^{+}, \mathrm{Mg}^{+}, \mathrm{Cl}$, fosfato, sulfato y bicarbonato (Villar, 2000). Investigaciones relacionadas al aumento de la concentración del $\mathrm{K}^{+}$en el cuerpo vítreo detectaron que no es influenciado por la temperatura ambiental después de la muerte (Komura \& Oshiro, 1997).

La investigación fue realizada en la ciudad de Quito, Ecuador, ubicada en la Cordillera de los Andes, a una altura de $2.850 \mathrm{~m}$ sobre el nivel del mar. En la revisión de literatura no se encontraron reportes de medición de estos parámetros ni de un estudio similar en el Ecuador. Lo anterior, llevó a investigar si existe un comportamiento similar o diferente respecto a las concentraciones de $\mathrm{Na}^{+}, \mathrm{Cl}^{-}$y Potasio $^{+}$en cuanto al IPM a la altura de Quito, y construir una base de datos que nos permitan aportar al conocimiento médico-legal. El objetivo de este trabajo fue establecer una correlación entre sodio $\left(\mathrm{Na}^{+}\right)$, cloro $\left(\mathrm{Cl}^{-}\right)$y potasio $\left(\mathrm{K}^{+}\right)$del cuerpo vítreo y el IPM, en cadáveres autopsiados en la Unidad de Medicina Legal Zona 9, Quito, Ecuador.

\section{MATERIAL Y MÉTODO}

Se diseñó un estudio longitudinal experimental que se llevó a cabo en la Unidad de Medicina Legal Zona 9 Quito, Ecuador, durante un período de cinco meses (octubre de 2019 a febrero de 2020).

Para la obtención de las muestras se consideraron los criterios de inclusión: hora de la muerte, la edad, etnicidad y la manera de la muerte. Se excluyeron los cadáveres con enfermedades infecto-contagiosas, con patología preexistente y en proceso avanzado de putrefacción. También se excluyeron a los menores y de la tercera edad, igualmente la etnia fue excluida por no haberse obtenido literatura referente.

Se utilizaron 128 muestras de humor vítreo del cuerpo vítreo provenientes de 16 autopsias. Los casos seleccionados fueron por muerte violenta (heridas por arma blanca, heridas por arma de fuego y tránsito); el fluido corporal se obtuvo de ambos bulbos oculares y se dividió para ocho alícuotas designadas a los IPM de 6, 12, 24, 48, 72, 96, 120 y 144 horas. Iniciando a la hora 6 como tiempo en que tarda el cadáver en llegar a la Unidad de Medicina Legal Zona 9, Quito.

Se excluyeron las muestras contaminadas con sangre o de aspecto turbio. La temperatura no fue considerada pues en la revisión de la literatura no se encontró que influya en el resultado, Quito no cuenta con temperaturas extremas y sus promedios oscilan entre 17 a $20^{\circ} \mathrm{C}$.

La toma de muestras se realizó mediante la técnica convencional descrita por Montefusco-Pereira \& Pinto, 2016, el humor vítreo se obtuvo por punción en el canto lateral de la esclera a 5-6 $\mathrm{mm}$ de distancia del limbo corneal, llegando al centro del bulbo ocular; se usaron jeringuillas estériles descartables de $10 \mathrm{ml}$ con aguja calibre $\mathrm{N}^{\circ} 21$, la aspiración de ambos bulbos oculares se realizó lentamente evitando contaminar la muestra con sangre o células. Posteriormente, se dividió el líquido extraido en 8 alícuotas, cada una de ellas, fue incorporada a conservación de $-20^{\circ} \mathrm{C}$ de cuerdo al IPM propuesto hasta su análisis.

La cuantificación de la concentración de electrolitos $\mathrm{Na}+, \mathrm{Cl}$ - y Potasio+ se realizó mediante analizador ISE de Roche Cobas (Roche Diagnostics). C501, calibrado para uroanálisis, y no fue necesaria la dilución. Durante la calibración y cada tres muestras, se midió un estándar interno para corregir los efectos de la desviación sistemática en la calibración. La trazabilidad el ISE compensador puede rastrearse hasta la fotometría de llama (sodio, potasio) y culombimetría (cloruro). 
El protocolo experimental fue aprobado por el Comité de Bioética de la Universidad San Francisco de Quito y se obtuvo el consentimiento informado del familiar.

Análisis estadístico. Para analizar la concentración de electrolitos, se calcularon las medias y la desviación estándar (DS), para cada uno de los ocho grupos que se establecieron según las horas que transcurrieron desde el momento de la muerte hasta las 144 horas. Se determinaron los intervalos de confianza al $95 \%$ de confianza (IC9 5\%) de la media para cada electrolito según los IPM establecidos. Se realizó la prueba de Kolmogorov-Smirnov, comprobándose que la misma no se distribuía de manera normal. Se realizó un análisis de correlación lineal utilizando el coeficiente de correlación de Spearman. Se consideró un valor de $\mathrm{p}$ menor a 0,05 como significativo. Los resultados se presentaron en forma tabular y gráfica. Se utilizó el SPSS versión 24 para el análisis de los datos.

\section{RESULTADOS}

Las concentraciones de los electrolitos vítreos $\mathrm{Na}^{+}$, $\mathrm{Cl}^{-}$y Potasio $^{+}$se encontraron incrementadas, presentando algunas variaciones durante el periodo postmortem estudiado. La Tabla I indica las concentraciones medias, los IC 95 $\%$ y la desviación estándar según grupos de IPM. Las medias del $\mathrm{Na}^{+}$y del Potasio ${ }^{+}$aumentaron en el tiempo, mientras que las concentraciones de $\mathrm{Cl}^{-}$se mantuvieron alrededor de la misma cifra mostrando un patrón irregular.

Las Fig. 1, 2 y 3 muestran las concentraciones de los electrolitos estudiados según avanzan las horas postmortem, poniendo en evidencia un aumento en el tiempo.

\section{Concentración media Sodio}

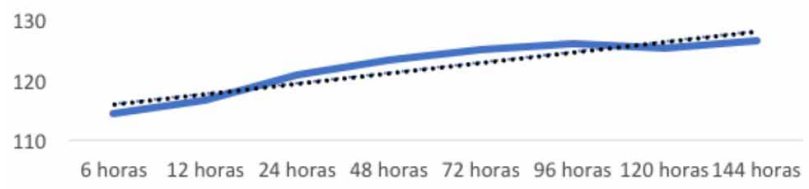

Fig. 1 Concentraciones medias de Sodio mmol/L según horas postmortem.

Tabla I. Concentraciones medias e intervalos de confianza al 95\% de los electrolitos estudiados según intervalos postmortem.

\begin{tabular}{|c|c|c|c|c|}
\hline & \multirow{2}{*}{$\begin{array}{c}\text { Media } \\
(\mathrm{mmol} / \mathrm{L})\end{array}$} & \multicolumn{2}{|c|}{ Intervalo de c onfianza al $95 \%$} & \multirow{2}{*}{$\begin{array}{c}\text { Desviación } \\
\text { estándar }(\mathrm{mmol} / \mathrm{L})\end{array}$} \\
\hline & & Inferior & Superior & \\
\hline \multicolumn{5}{|l|}{ Sodio } \\
\hline 6 horas & 114,1 & 104,2 & 124,1 & 20,3 \\
\hline 12 horas & 110,5 & 96,8 & 124,2 & 27,9 \\
\hline 24 horas & 120,7 & 113,8 & 127,7 & 14,2 \\
\hline 48 horas & 123,1 & 116,3 & 130,0 & 13,9 \\
\hline 72 horas & 124,8 & 120,2 & 129,5 & 9,5 \\
\hline 96 horas & 125,9 & 120,9 & 131,0 & 10,3 \\
\hline 120 horas & 125,2 & 120,2 & 130,3 & 10,3 \\
\hline 144 horas & 126,2 & 121,1 & 131,4 & 10,4 \\
\hline \multicolumn{5}{|l|}{ Cloro } \\
\hline 6 horas & 100,4 & 92,5 & 108,4 & 16,2 \\
\hline 12 horas & 94,4 & 83,8 & 105,0 & 21,65 \\
\hline 24 horas & 99,5 & 92,9 & 106,3 & 13,7 \\
\hline 48 horas & 101,0 & 91,0 & 111,2 & 20,5 \\
\hline 72 horas & 104,2 & 99,8 & 108,6 & 8,9 \\
\hline 96 horas & 101,2 & 91,0 & 111,2 & 24,8 \\
\hline 120 horas & 98,6 & 86,4 & 110,9 & 25,0 \\
\hline 144 horas & 99,5 & 87,3 & 111,9 & 16,2 \\
\hline \multicolumn{5}{|l|}{ Potasio } \\
\hline 6 horas & 5,4 & 5,0 & 5,9 & 0,9 \\
\hline 12 horas & 5,7 & 4,9 & 6,6 & 1,6 \\
\hline 24 horas & 6,3 & 5,6 & 7,2 & 1,6 \\
\hline 48 horas & 6,4 & 5,6 & 7,3 & 1,7 \\
\hline 72 horas & 6,9 & 5,7 & 8,2 & 2,5 \\
\hline 96 horas & 7,4 & 6,0 & 8,9 & 2,8 \\
\hline 120 horas & 7,9 & 5,8 & 10,1 & 4,3 \\
\hline 144 horas & 7,9 & 5,9 & 10,1 & 4,3 \\
\hline
\end{tabular}




\section{Concentración media Cloro}

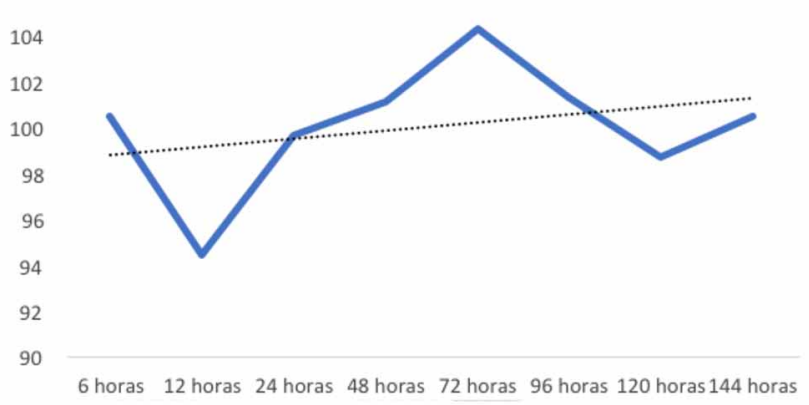

Fig. 2 Concentraciones medias de Cloro mmol/L según horas postmortem.

\section{Concentración media Potasio}

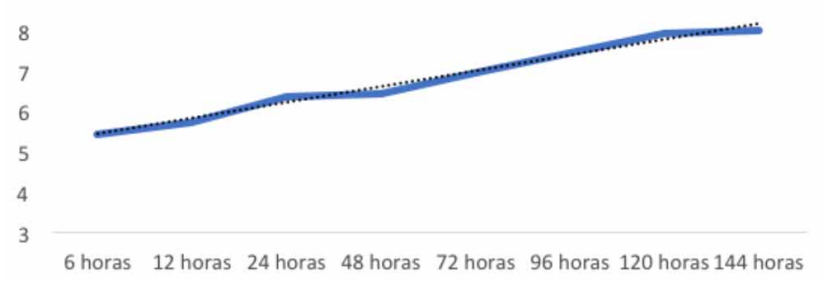

Fig. 3. Concentraciones medias de Potasio mmol/L según horas postmortem.

La Tabla II demuestra la correlación estadísticamente significativa entre las horas postmortem y las concentraciones del Sodio $\left(\mathrm{Na}^{+}\right)$, Cloro $\left(\mathrm{Cl}^{-}\right)$y Potasio $\left(\mathrm{K}^{+}\right)$estudiados

Tabla II. Correlación entre horas postmortem y concentración de los electrolitos estudiados.

\begin{tabular}{|c|c|c|}
\hline & $\begin{array}{l}\text { Coeficiente de correlación Rho } \\
\text { de Spearman }\end{array}$ & Valor de $p$ \\
\hline Sodio & 0,22 & 0,01 \\
\hline Cloro & 0,45 & 0,00 \\
\hline Potasio & 0,36 & 0,00 \\
\hline
\end{tabular}

\section{DISCUSIÓN}

El presente estudio muestra que la concentración de los electrolitos estudiados posee una tendencia a aumentar en el tiempo; sin embargo, y aún con una ligera tendencia al aumento, la concentración del Cl- presentó un patrón irregular. Se corroboró además, que a medida que aumentaron las horas después del fallecimiento, también aumentaron las concentraciones de forma lineal de manera estadísticamente significativa.
El humor vítreo del cuerpo vítreo es menos susceptible que otros fluidos a cambios químicos y a la contaminación (Madea et al., 1989; Ahi, R. S. \& Garg, 2011; Patel et al., 2016), se considera de fácil acceso y su composición es bastante similar al fluido acuoso, al líquido cerebroespinal y al suero (Ahi, R. S. \& Garg, 2011).

Para autores como Jashnani et al. (2010) el $\mathrm{Cl}^{-}$no tiene ningún rol en la estimación del IPM; en nuestro estudio su comportamiento fue irregular, aunque se demostró una correlación lineal. A pesar de este resultado, los autores consideran que no es recomendable su utilización para estimación del IPM.

Varios investigadores como Cañadas \& Gisbert Calabuig (2018) señalaron que el $\mathrm{Na}^{+}$es estable en las primeras $20 \mathrm{~h}$ postmortem y después tiende a ascender; Jashnani et al. (2010) encontraron una relación inversamente proporcional al IPM entre el $\mathrm{Na}^{+}$y el $\mathrm{K}^{+}$. En esta investigación las concentraciones del $\mathrm{Na}^{+}$presentaron tendencia al aumento en todos los IPM analizados hasta las $144 \mathrm{~h}$. Al igual que Naumann (1959), Jashnani et al. (2010) y Cañadas \& Gisbert Calabuig (2018) quienes obtuvieron concentraciones como $118-152 \mathrm{mEq} / \mathrm{l}, 133-137 \pm 10.45$ y $149 \mathrm{mEq} / \mathrm{l}$ respectivamente, en esta investigación se detectó una media similar de 114,1 -126, 1mmol/l. El nivel normal del $\mathrm{K}^{+}$en el humor vítreo es aproximadamente 3,8mmol/1 (Ahi, R. S. \& Garg, 2011), siendo adecuado para muchos análisis y para estimar el IPM (Ahi \& Garg, 2011; Bartolote et al.,2011; Cañadas \& Gisbert Calabuig, 2018).

Es necesario señalar que varias ecuaciones han sido descritas en la literatura que relacionan al $\mathrm{K}^{+}$vítreo con el IPM y muchas están basadas en el modelo de regresión lineal del $\mathrm{K}^{+}$postmortem que aumenta proporcionalmente al tiempo (Jashnani et al., 2010; Bartolote et al.,2011).

Para determinar el IPM, Sturner, W. Q. \& Gantner Jr, G. E. (1964) desarrollaron una fórmula citada ampliamente: IPM (horas) $=\left(7.14\right.$ x K $\left.\mathrm{K}^{+}\right)-39.1$ (Coe, 1989 ; Mihailovic et al., 2012). La desventaja de ella fue una pendiente plana y la diferencia significativa entre el potasio real y el estimado (Jashnani et al., 2010; Mihailovic et al., 2012).

Ortmann et al. (2016) encontraron relación lineal entre $\mathrm{K}^{+}$vítreo y el IPM que no es línea recta sino bifásica. Los resultados del presente estudio demuestran una relación en forma de línea recta entre las horas postmortem y las concentraciones del $\mathrm{K}^{+}$vítreo, pero no se demostró una recta bifásica.

Patel et al. (2016) observaron en su estudio que por cada 12 horas después de la muerte se incrementaban aproxi- 
madamente $2 \mathrm{mmol} / 1$ de concentración de $\mathrm{K}^{+}$en el humor vítreo, sigue la regla del 12. En el presente estudio se pudo comprobar también un aumento sostenido de la concentración de este electrolito en el tiempo con resultados similares a los obtenidos por estos autores.

En esta investigación se corroboró que existe una correlación lineal entre el IPM y el aumento del $\mathrm{K}^{+}$descrito por autores como (Coe, 1989; Jashnani et al., 2010; Swain et al., 2015; Ding et al., 2017; Yang et al., 2018) quienes también describieron esta relación pero con dispersión creciente pasados las $20 \mathrm{~h}$ postmortem. En otros estudios también se encontró una relación lineal entre 12 y 100 horas postmortem, Ahi \& Garg (2011) Madea et al. (1989) encontraron que las concentraciones de $\mathrm{Na}^{+}, \mathrm{Ca}, \mathrm{Cl}^{-}$y la urea fueron estables en el intervalo postmortem de $120 \mathrm{~h}$.

En contraste con los estudios previos que describen una relación no lineal entre el $\mathrm{K}^{+}$vítreo y el IPM, esto proporciona una mejor estimación del IPM especialmente en periodos postmortem más largos (Zilg et al., 2015).

Madea et al. (1989) crearon una fórmula utilizando una pendiente más pronunciada, que evitaría la sobreestimación sistemática que ocurre en el caso de las pendientes menos pronunciadas. Otros investigadores analizaron la concentración del $\mathrm{Ca}, \mathrm{Mg}, \mathrm{Na}^{+}$, hipoxantina y amonio en el humor vítreo, con el objetivo de lograr mayor confiabilidad, además utilizaron análisis de datos multivariados (Bartolote et al., 2011; Chandrakanth et al., 2013).

El aumento relativo de potasio se ve afectado por las temperaturas altas y la exposición del cadáver a esas temperaturas. Un cadáver dejado a temperatura ambiente o superior durante un tiempo considerable también progresará rápidamente a través de las etapas de descomposición y complicará aún más la capacidad de utilizar el humor vítreo para las pruebas (McCleskey et al., 2016). El estudio de Foster $e t$ al. (2016) no consideró la temperatura y fue referido como una limitación del estudio, y supone que esta variable podría tener efectos en los resultados. En este estudio la temperatura no fue considerada por haberse desarrollado en un ciudad de ambiente tropical, donde no existen cambios extremos en la temperatura ambiental. Sin embargo, en futuros estudios se podría considerar a la temperatura como variable a investigar.

Foster et al. (2016) consideraron a la edad como otro factor de confusión; sin embargo, no confirmaron una relación significativa entre la edad y el nivel de potasio en el humor vítreo, al no encontrar significancia entre la edad y el nivel de $\mathrm{K}^{+}$, en este estudio no se consideró esta variable.

\section{CONCLUSIÓN}

El estudio demostró que los elementos bioquímicos del humor vítreo como el $\mathrm{Na}^{+}, \mathrm{Cl}^{-}$y $\mathrm{K}^{+}$, en particular el $\mathrm{K}^{+}$ como variable dependiente en el análisis de regresión lineal mostró un aumento considerable con el transcurso del tiempo postmortem. El Na+ mantuvo tendencia al aumento mientras el comportamiento del $\mathrm{Cl}^{-}$fue irregular con ligera tendencia al aumento, esto hace del método bioquímico una herramienta confiable para estimar el IPM. Los resultados son similares a los obtenidos por otros investigadores por lo que se asume que la altura no influye en la relación de los electrolitos estudiados y el IPM.

\section{AGRADECIMIENTOS}

Nuestro agradecimiento al Hospital Quito $\mathrm{N}^{\circ} 1$ Policía Nacional del Ecuador, y a la Dra. Dolores Maldonado Jefa del Laboratorio Clínico, por su espíritu progresista y colaboración con la investigación científica, en búsqueda de mejorar métodos que aportarán con la investigación penal y con las incógnitas planteadas por los investigadores judiciales, policiales y por los familiares de los cadáveres encontrados.

GUERRERO-URBINA, C.; FORS, M.; DEL SOL, M. \& FONSECA, G. M. Correlation between sodium, chlorine and potassium of the vitreous body and the postmortem interval above 2,850 msnm. Int. J. Morphol., 39 (2):554559,2021

SUMMARY: The postmortem interval (PMI) is an important challenge to be solved in forensic pathology, and it consists of being able to determine the time elapsed from death to the moment of autopsy. Given the unreliability of some methods due to the great influence of external factors and those specific to the corpse, forensic biochemistry has received considerable attention for its levels of safety. The city of Quito is located in the Andes mountain range at 2850 meters above sea level, however, in the literature there are no reports of measurement of these parameters at a height like this. The objective was to establish a correlation between sodium $\left(\mathrm{Na}^{+}\right)$, chlorine $\left(\mathrm{Cl}^{-}\right)$and potassium $\left(\mathrm{K}^{+}\right)$of the vitreous humor and the MPI at a height of 2,850 masl. For the study, 128 samples of vitreous humor were used from 16 autopsies performed in the Zone 9 Legal Medicine Unit, in IPM of 6, 12, 24, 48, 72, 96, 120 and 144 hours. To obtain the vitreous humor, the conventional method was followed, it was divided for the 8 aliquots and it was frozen at $-20^{\circ} \mathrm{C}$ until the moment of its analysis. The quantification of the 
concentration of electrolytes $\mathrm{Na}^{+}, \mathrm{Cl}-$ and $\mathrm{K}^{+}$was carried out using an ISE analyzer from Roche Cobas (Roche Diagnostics) C501, calibrated for urinalysis, and no dilution was necessary. During calibration and every third sample, an internal standard was measured to correct for the effects of systematic deviation on the calibration. The statistical approach was based on a linear correlation analysis, using the Spearman correlation coefficient. The correlation between the postmortem hours and the concentrations of the different electrolytes were statistically significant. A significant linear correlation between the PMI and the increase in $\mathrm{K}^{+}$in vitreous humor could be corroborated.

\section{Vitreous body.}

KEY WORDS: Electrolytes; Postmortem interval;

\section{REFERENCIAS BIBLIOGRÁFICAS}

Ahi, R. S. \& Garg, V. Role of vitreous potassium level in estimating postmortem interval and the factors affecting it. Journal of clinical and diagnostic research., 5(1): 13-15, 2011.

Bortolotti, F.; Pascali, J. P.; Davis, G. G.; Smith, F. P.; Brissie, R. M. \& Tagliaro, F. Study of vitreous potassium correlation with time since death in the postmortem range from 2 to 110 hours using capillary ion analysis. Medicine, Science and the Law., 51(1_suppl): 20-23, 2011.

Cañadas, E. V. Gisbert Calabuig. Medicina Legal Y Toxicológica. Elsevier, 2018.

Chandrakanth, H. V.; Kanchan, T.; Balaraj, B. M.; Virupaksha, H. S. \& Chandrashekar, T. N. Postmortem vitreous chemistry-an evaluation of sodium, potassium and chloride levels in estimation of time since death (during the first $36 \mathrm{~h}$ after death). Journal of forensic and legal medicine., 20(4): 211-216, 2013.

Coe, J. I. Vitreous potassium as a measure of the postmortem interval: an historical review and critical evaluation. Forensic science international., 42(3): 201-213, 1989.

Ding, Y.; Li, X.; Guo, Y.; Duan, W.; Ling, J.; Zha, L. \& Cai, J. Estimation

* of postmortem interval by vitreous potassium evaluation with a novel fluorescence aptasensor. Scientific reports., 7(1): 1-9, 2017.

Donaldson, A. E. \& Lamont, I. L. Estimation of post-mortem interval using biochemical markers. Australian Journal of Forensic Sciences., 46(1): 8-26, 2014.

Foster, S. N.; Smith, P. R.; Biggs, M.; Rutty, G. N.; Hollingbury, F. E. \& Morley, S. R. Estimation of postmortem interval using vitreous potassium levels in cases of fatal road traffic collision. Archiwum Medycyny Sa $\mathrm{c}_{c}$ dowej i Kryminologii/Archives of Forensic Medicine and Criminology., 66(2): 71-82, 2016.

Gerometta, R.; Larroza, G. O.; Pimpinella, P. \& Genero, S. Variación de la Presión intraocular en función del tiempo: contribución a la determinación del verdadero intervalo de muerte (VIM). Revista Mexicana De Medicina Forense y Ciencias De La Salud., 4(1): 2019.

Jashnani, K. D.; Kale, S. A. \& Rupani, A. B. Vitreous humor: biochemical constituents in estimation of postmortem interval. Journal of forensic sciences., 55(6): 1523-1527, 2010.

Kokavec, J.; Min, S. H.; Tan, M. H.; Gilhotra, J. S.; Newland, H. S.; Durkin, S. R. \& Casson, R. J. Biochemical analysis of the living human vitreous. Clinical \& experimental ophthalmology., 44(7): 597-609, 2016

Komura, S. \& Oshiro, S. Potassium levels in the aqueous and vitreous humor after death. The Tohoku journal of experimental medicine., 122(1): 65-68, 1977.
Li, W. Chang, Y.; Cheng, Z.; Ling, J.; Han, L.; Li, X. \& Ding, Y. Vitreous humor: A review of biochemical constituents in postmortem interval estimation. Journal of Forensic Science and Medicine., 4(2): 85, 2018.

Madea, B. Methods for determining time of death. Forensic science, medicine, and pathology., 12(4): 451-485, 2016.

Madea, B.; Henssge, C.; Hönig, W. \& Gerbracht, A. References for determining the time of death by potassium in vitreous humor. Forensic science international., 40(3): 231-243, 1989.

McCleskey, B. C.; Dye, D. W. \& Davis, G. G. Review of Postmortem Interval Estimation Using Vitreous Humor: Past, Present, and Future. Academic forensic pathology., 6(1): 12-18, 2016.

Mihailovic, Z.; Atanasijevic, T.; Popovic, V.; Milosevic, M. B. \& Sperhake, J. P. Estimation of the postmortem interval by analyzing potassium in the vitreous humor: could repetitive sampling enhance accuracy? The American journal of forensic medicine and pathology., 33(4): 400-403, 2012.

Montefusco-Pereira, C. V. \& Pinto, L. D. M. A. El humor vítreo como fluido biológico de importancia clínica en ciencias forenses. Acta bioquímica clínica latinoamericana., 50(1): 27-35, 2016.

Moore, K. L. \& Dalley, A. F. Anatomía con orientación clínica. 7 a ed. Barcelona, Ed. Médica Panamericana, 2013.

Mulla, A.; Massey, K. L. \& Kalra, J. Vitreous humor biochemical constituents: evaluation of between-eye differences. The American journal of forensic medicine and pathology., 26(2): 146-149, 2005.

Ortmann, J.; Markwerth, P. \& Madea, B. Precision of estimating the time since death by vitreous potassium-Comparison of 5 different equations. Forensic science international., 269: 1-7, 2016.

Patel, U. P.; Patel, J.; Prajapati, P. \& Govekar, G. A Study to Evaluate of Time Since Death From Potassium Level of Vitreous Humour. Natl J Med Res., 6(3): 255-8, 2016.

Sturner, W. Q. \& Gantner Jr, G. E. The postmortem interval. American journal of clinical pathology., 42(2): 137-144, 1964.

Tagliaro, F.; Manetto, G.; Cittadini, F.; Marchetti, D.; Bortolotti, F. \& Marigo, M. Capillary zone electrophoresis of potassium in human vitreous humour: validation of a new method. Journal of Chromatography B: Biomedical Sciences and Applications., 733(1-2): 273-279, 1999.

Thierauf, A.; Musshoff, F. \& Madea, B. Post-mortem biochemical investigations of vitreous humor. Forensic science international., 192(13): 78-82, 2009.

Villar, D. F. Anatomía Ocular, 2000.

Yang, M.; Li, H.; Yang, T.; Ding, Z.; Wu, S.; Qiu, X. \& Liu, Q. A study on the estimation of postmortem interval based on environmental temperature and concentrations of substance in vitreous humor. Journal of forensic sciences., 63(3): 745-751, 2018

Zhou, B.; Zhang, L.; Zhang, G.; Zhang, X. \& Jiang, X. The determination of potassium concentration in vitreous humor by low pressure ion chromatography and its application in the estimation of postmortem interval. Journal of Chromatography B., 852(1-2): 278-281, 2007.

Zilg, B.; Bernard, S.; Alkass, K.; Berg, S. \& Druid, H. A new model for the estimation of time of death from vitreous potassium levels corrected for age and temperature. Forensic science international., 254: 158-166, 2015.

\section{Dirección para correspondencia: \\ Dr. Gabriel Fonseca \\ Facultad de Odontología \\ Universidad de La Frontera \\ Temuco - CHILE}

\section{E-mail: gabriel.fonseca@ufrontera.cl}

Recibido: 17-11-2020

Aceptado: 26-01-2021 